\title{
Alignment of valence photoemission, x-ray absorption, and substrate density of states for an adsorbate on a semiconductor surface
}

\author{
J. Schnadt, ${ }^{1, *}$ J. N. O’Shea, ${ }^{2}$ L. Patthey, ${ }^{3}$ J. Krempaský, ${ }^{3}$ N. Mårtensson, ${ }^{4,1}$ and P. A. Brühwiler ${ }^{1, \dagger}$ \\ ${ }^{1}$ Department of Physics, Uppsala University, Box 530, 75121 Uppsala, Sweden \\ ${ }^{2}$ School of Physics and Astronomy, University of Nottingham, Nottingham NH7 2RD, United Kingdom \\ ${ }^{3}$ Swiss Light Source, Paul Scherrer Institut, 5232 Villigen-PSI, Switzerland \\ ${ }^{4}$ MAX-Lab, University of Lund, Box 118, 22100 Lund, Sweden \\ (Received 21 February 2003; published 26 June 2003)
}

\begin{abstract}
A method for determining the placement of x-ray absorption data into an experimental density of states for an adsorbate-semiconductor system is motivated and illustrated with the cases of bi-isonicotinic acid, isonicotinic acid, and benzoic acid on rutile $\mathrm{TiO}_{2}(110)$. Special attention is paid to experimental aspects, as well as the role of $Z+1$ effects and the choice of reference level.
\end{abstract}

DOI: 10.1103/PhysRevB.67.235420 PACS number(s): 73.20.Hb, 78.70.Dm, 79.60.Dp, 73.20.Jc

\section{INTRODUCTION}

Heterogeneous systems are interesting because of the level of complexity they display compared to simple crystals and because they give one flexibility to tune properties by combining systems with different attractive features. Our motivation here is nanostructured systems such as the Grätzel cell (see, e.g., Ref. 1), which can be loosely described as consisting of light-absorbing molecules coupled to a highsurface-area semiconducting charge separator, but the problem can be generalized to apply to many heterogeneous systems whose electronic properties are of interest. ${ }^{2}$ A full characterization of the electronic excitations of an adsorbate or other coupled system requires the assessment of both the occupied and the unoccupied electronic states of the coupled components. The occupied states are conveniently mapped with photoemission spectroscopy (PES), and the unoccupied states can be measured with methods like inverse photoemission (IPES) and x-ray absorption spectroscopy (XAS). Of the latter two, IPES has a very low cross section and is not generally accessible. XAS presents interpretational challenges due to the presence of a core hole, which because of its localized extent tends to strongly perturb the valence states much in the way that an extra nuclear charge would do $(Z+1$ effect). XAS has the advantage that it is available at many synchrotron beamlines and can therefore be applied to the same samples as PES, which facilitates the comparison of data from the two spectroscopies, as well as providing an atom-specific probe. To exploit the full potential of the methods, it is useful to directly compare PES, which measures electron kinetic energies, and XAS, which measures photon energies necessary to excite core electrons to unoccupied levels.

For molecules chemisorbed on metal surfaces it has been shown that a common energy scale for both PES and XAS is readily established by referring the binding energy of the core level relevant for XAS to the metal Fermi level $\left(E_{\mathrm{F}}\right)$. For these systems the onset of states in XAS corresponds to that particular energy. ${ }^{3,4}$ This result relies on the efficient charge transfer screening provided in these systems and thus cannot readily be applied to adsorbates on semiconducting substrates. Moreover, in general no spectral structure is associated with the chemical potential $\left(E_{\mathrm{F}}\right)$ in the latter cases, and sample charging can shift the photoemission spectra by unknown amounts. Hence both intrinsic and practical aspects make the analysis of semiconducting substrates more complicated than that of metallic substrates.

Here we will detail and illustrate a strategy, 2,5 similar to the one for metallic samples, for the case of an adsorbate on a semiconductor surface. ${ }^{6}$ This strategy relates the energies measured in PES and XAS, and the substrate density of states is included in the full image of the electronic structure. The relationship between the latter and the core-excited adsorbate density of states measured in XAS is particularly interesting for charge transfer studies involving core-excited adsorbate states, since it indicates from which of these states such a transfer is possible., ${ }^{2,5}$ As will be discussed below, results in that matter should also have some bearing on the elucidation of the charge transfer characteristics of optically excited adsorbates. We begin with the case of bi-isonicotinic acid $\left[\left(\mathrm{C}_{5} \mathrm{H}_{3} \mathrm{NCOOH}\right)_{2}\right]$ adsorbed on rutile $\mathrm{TiO}_{2}(110)$, with which the alignment procedure is presented in some detail and for which several useful auxiliary investigations have already been carried out. We then apply the procedure to isonicotinic acid $\left(\mathrm{C}_{5} \mathrm{H}_{4} \mathrm{NCOOH}\right)$ on $\mathrm{TiO}_{2}(110)$ and benzoic acid $\left(\mathrm{C}_{6} \mathrm{H}_{5} \mathrm{COOH}\right)$ on $\mathrm{TiO}_{2}(110)$. The latter comparison provides additional insight into core hole effects, constituting an experimental investigation of the $Z+1$ effect.

\section{EXPERIMENT}

The PES and $\mathrm{N} 1 s$ and $\mathrm{C} 1 s$ XAS experiments were performed at beamline 22/D1011 at MAX-Lab in Lund. ${ }^{10,11}$ The complete experimental details, including the characterization of the samples, are contained in Refs. 7 (biisonicotinic acid), 12, and 13 (isonicotinic acid and benzoic acid).

In short, the base pressure was in the low- $10^{-10}$-Torr range in the preparation chamber and in the low- $10^{-11}$-Torr range in the analysis chamber. The $\mathrm{TiO}_{2}(110)$ single crystals, purchased from Djévahirdjian, Industrie de Pierre Scientifique, Monthey, Switzerland, and from MaTecK, Jülich, Germany, were made conducting by annealing in 


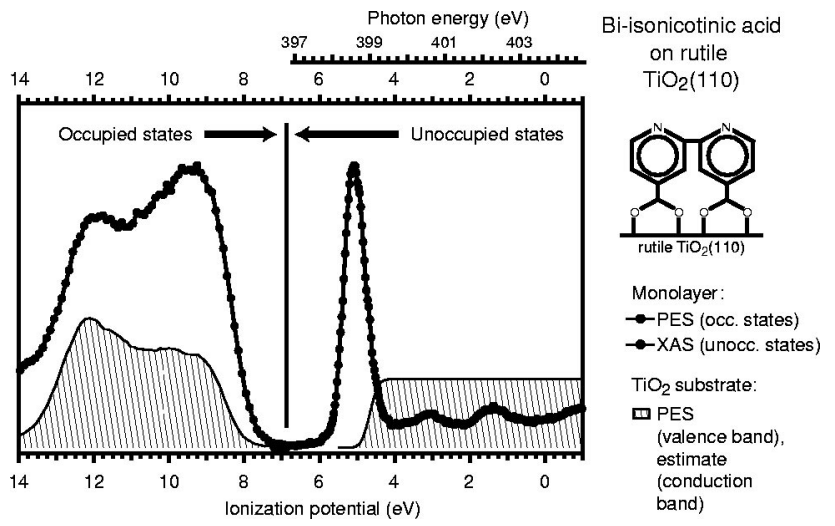

FIG. 1. Combination of valence photoemission spectra (PES), an X-ray absorption spectrum (XAS), and a substrate band gap estimate for a monolayer of bi-isonicotinic acid adsorbed on rutile $\mathrm{TiO}_{2}(110)$ to give an image of the electronic structure of the coreexcited adsorbate system. The spectra have been placed on a common energy scale; in addition, the photon energy scale of the x-ray absorption spectrum has been given.

$1 \times 10^{-6}$ Torr $\mathrm{O}_{2}$ at $700{ }^{\circ} \mathrm{C}$. The substrate surface was then prepared by repeated cycles of Ar sputtering and annealing in $1 \times 10^{-6}$ Torr $\mathrm{O}_{2}$ at a temperature between 570 and $700^{\circ} \mathrm{C}$, until no spectral signatures of $\mathrm{C}$ and $\mathrm{O}$ contaminations were found. The chemicals were obtained from Sigma-Aldrich with a guaranteed purity of more than $99 \%$ and were thoroughly outgassed in vacuum before use. The molecules were deposited onto the substrate from a thermal sublimator with pressure and temperature monitors. By keeping the substrate at $200^{\circ} \mathrm{C}$ it was achieved that either close to a monolayer or a full monolayer of adsorbates was formed.

The x-ray absorption spectra were measured in partial yield mode employing detector biases of $-330 \mathrm{~V}(\mathrm{~N} 1 s)$ and $-220 \mathrm{~V}(\mathrm{C} 1 s)$, respectively. The C $1 s$ x-ray absorption spectrum had to be corrected for a highly varied background, which in our case is mainly due to beamline-induced changes in the second-order light transmission. ${ }^{14}$

The monolayers that result from this procedure are characterized by a $2 \mathrm{M}$-bidentate-adsorbate-substrate bond between each carboxylic group and two Ti atoms of the surface. The pyridine (isonicotinic acid), bipyridine (biisonicotinic acid), and phenyl (benzoic acid) rings stand upright on the surface with an approximate tilt angle of $25^{\circ}$ (bi-isonicotinic acid) (Ref. 7) and an estimated maximum tilt angle of $40^{\circ}$ (isonicotinic and benzoic acids) (Ref. 12). For the monomers adsorbate-adsorbate interactions are significant, as detailed in Refs. 12 and 13.

\section{RESULTS AND DISCUSSION}

In Fig. 1 the valence electronic structure of bi-isonicotinic acid on $\mathrm{TiO}_{2}(110)$ is illustrated. Bi-isonicotinic acid is a molecule that is important as a ligand in the "N3" dye employed for light absorption sensitization of oxide substrates. ${ }^{15}$ In order to understand the electron transfer from an excited adsorbate molecule to the oxide substrate knowledge of the valence electronic structure is imperative, since such a transfer on the fs time scale can proceed only if the substrate

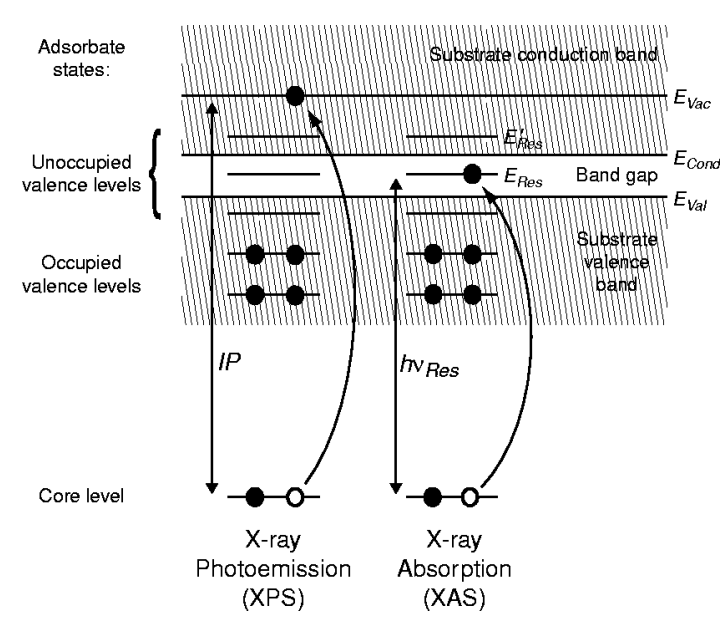

FIG. 2. Schematic of the energetics of an adsorbate system on a semiconductor surface (in the spirit of Ref. 2). The adsorbate is assumed to retain its molecular character in spite of the substrate coupling, and thus its energy levels are discrete, albeit often broadened by the bonding. The semiconductor continua are defined by the hatched areas, while the gap is not patterned. Important in this context is that the discrete molecular levels correspond to the coreexcited adsorbate system, while the substrate energy continua should refer to quasiparticle states.

density of states matches that of the adsorbate. For semiconductor systems this means that one has to be able to elucidate the relative positions of the band gap and adsorbate states. A combination of PES, XAS, and knowledge of the quasiparticle band gap yields the desired parameters for a core-excited state, as can be seen from Fig. 1 for bi-isonicotinic acid on $\mathrm{TiO}_{2}(110)$. The lowest $\pi$ resonance of the adsorbate lies approximately $2.5 \mathrm{eV}$ above the valence-band edge-i.e., an estimated $0.6 \mathrm{eV}$ below the substrate conduction-band edge, which has been placed $3.1 \mathrm{eV}$ (the value for the optical band gap) above the valence-band edge for rutile $\mathrm{TiO}_{2}$. Thus, any charge transfer from this particular resonance is prevented (within the core-hole lifetime) by the electronic mismatch of adsorbate and substrate states. In contrast, the two higher resonances in the $\mathrm{x}$-ray absorption spectrum overlap the substrate density of states and a rapid charge transfer is not only possible, but also observed. ${ }^{5}$

Before further describing how the spectra obtained from the different techniques and the substrate density of states can be placed on a common energy scale, we would like to start out with a consideration of the relationship between $\mathrm{X}$-ray absorption and photoemission for the case of an adsorbate on a semiconductor surface. The corresponding energetics are presented in Fig. 2. As pointed out in the Introduction, no spectral structure is in general connected to the chemical potential for the case of systems with electronic gaps (insulators and semiconductors). Therefore it is more convenient to use the vacuum level as a reference for the photoemission spectra since it can be obtained routinely. ${ }^{8}$ The resulting electron removal energies are often termed ionization potentials (IPs), and their values are those of excitations in which the electron is promoted from a core or valence level to the vacuum level. This is also the energy necessary to ionize the sample, containing $N$ electrons, to the ground state of the 

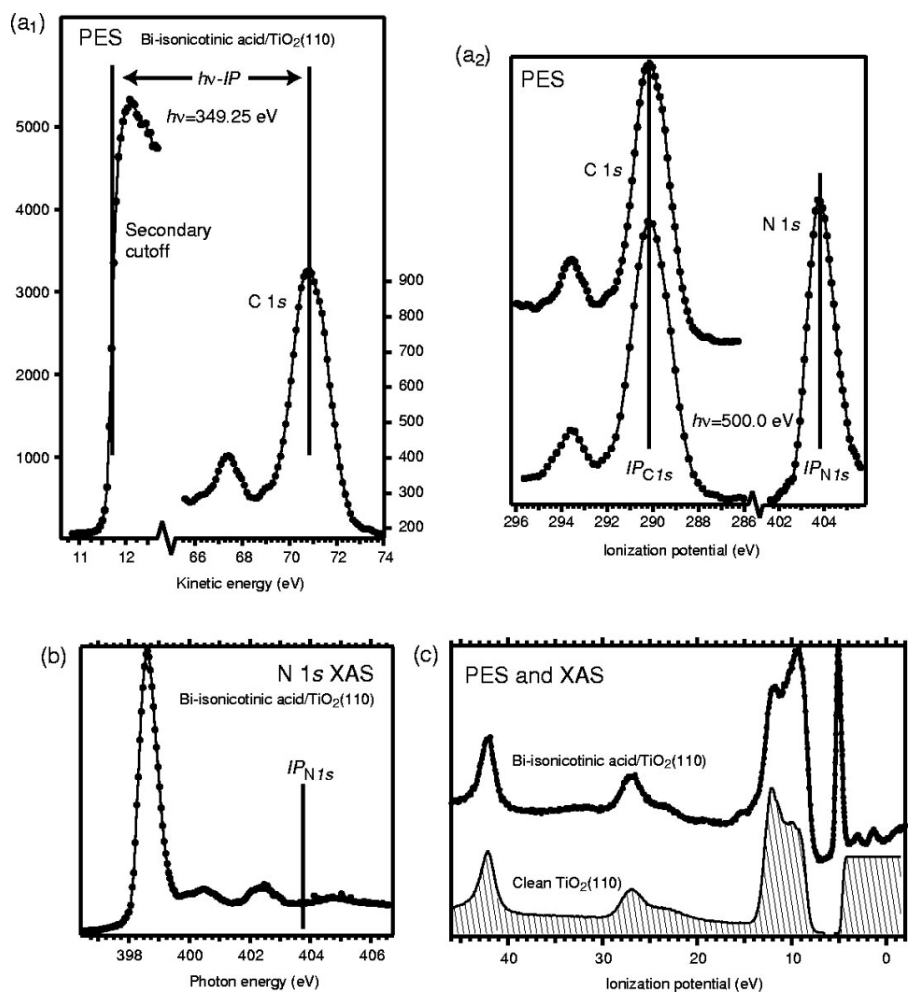

FIG. 3. Illustration of the fundamental calibration and alignment procedure used here. $\left(a_{1}\right)$ The ionization potential of the core levels is derived from the separation of the core level from the secondary electron cutoff. This energy has been taken to lie halfway up the cutoff curve. $\left(\mathrm{a}_{2}\right)$ In the present case, the so-calibrated C $1 s$ core level was used to determine the ionization potential of the other core level. (b) The photon energy in the x-ray absorption spectrum that numerically equals the value of the ionization potential corresponds to the vacuum level. By setting it to zero and inverting the energy scale the x-ray absorption spectrum is placed on the same scale as the photoemission spectra. (c) Upper curve: combination of the monolayer valence photoemission and x-ray absorption spectra, aligned as described in the text. Lower curve: the substrate density of states is taken into account by a valence photoemission spectrum of the clean crystal (approximately $7-46 \mathrm{eV}$ ionization potential) and by an estimate of the location of the substrate conduction band (approximately $-2-6 \mathrm{eV}$ ).

system containing a vacancy in the particular orbital probe and with $N-1$ remaining electrons. ${ }^{16}$ Now the x-ray absorption spectra can be placed on the same scale by the observation that an excitation to the vacuum level in PES is also measured at a particular point in the x-ray absorption spectrum, with the photon energy given by the ionization potential. The common scale is then established by identifying the photon energy nominally equal to the corresponding corelevel ionization potential, choosing this energy as the XAS origin and inverting the relative energies. This corresponds to determining electron removal energies in XAS. In fact, any other photoemission reference level (such as the conductionband edge, the valence-band edge, or the Fermi edge, if accessible) is equally usable, as long as the relative energies of the valence and core levels are known. ${ }^{2}$ This can be appreciated by assuming that not the ionization potential, but a somewhat higher (lower) energy had been chosen as reference. If the energies of the valence band are known relative to the core level, a prerequisite in the procedure, these energies are then moved to higher (lower) values. But also the reference point of the $\mathrm{x}$-ray absorption spectrum is changed by the new choice and corresponds to a higher (lower) photon energy, which moves the x-ray absorption spectrum features to lower (higher) energies on the common scale. The relationship between XAS and PES is thus conserved.
In Fig. 3 these concepts are now applied to bi-isonicotinic acid on $\mathrm{TiO}_{2}(110)$ to show how the full picture of the valence electronic structure presented in Fig. 1 emerges. The determination of the ionization potentials follows the procedure outlined in Ref. 8 and is described again in the following. It should be stressed that this procedure handles shifts that are connected with sample charging. ${ }^{17}$

In panel $\left(\mathrm{a}_{1}\right)$ the secondary cutoff and the $\mathrm{C} 1 \mathrm{~s}$ level of a bi-isonicotinic acid monolayer on rutile $\mathrm{TiO}_{2}(110)$ are shown, one measured directly measured after the other without any intervening change in the experimental setup. ${ }^{18}$ The crystal was biased by $-7.0 \mathrm{~V}$ to ensure that the low-kineticenergy electrons would reach the analyzer. Thus the kinetic energies shown in Fig. 3 are shifted to higher values. The photon energy for this measurement is calibrated by measuring the kinetic energy difference of a photoemission feature excited with light in first and second order at the chosen monochromator setting. This, combined with the observed kinetic energy, enables one to determine the ionization potential. The scales for the ionization potentials of the other core levels and the valence band are obtained either in the same way or, as in the present case, by using the $\mathrm{C} 1 s$ as a reference [see Fig. 3( $\left.\mathrm{a}_{2}\right)$ ].

In panel (b), the N $1 s$ absorption spectrum is given. First, 


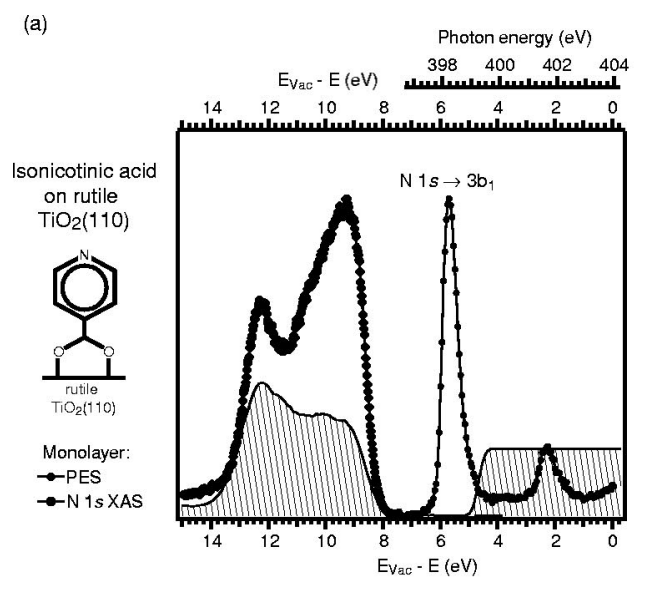

(b)

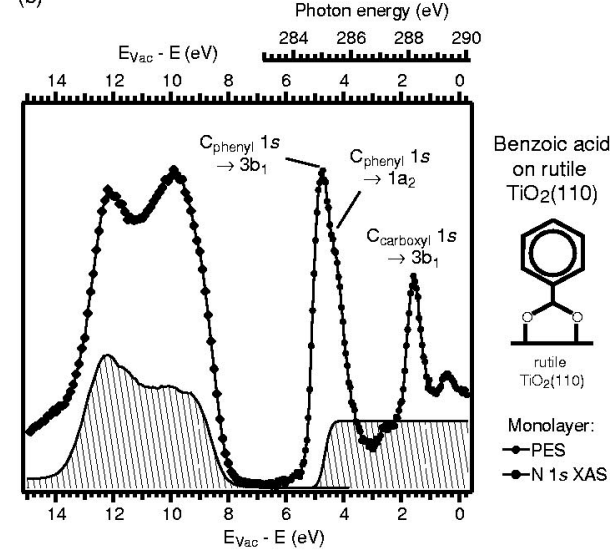

(c)

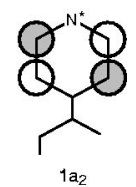

$1 a_{2}$

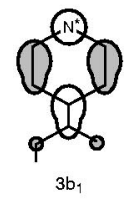

$\mathrm{TiO}_{2}$ substrate: $⿴ \mathrm{PES}$ (valence band), estimate (conduction band)

FIG. 4. Experimental density of states for isonicotinic acid and benzoic acid on $\mathrm{TiO}_{2}(110)$. (a) Isonicotinic acid. As for bi-isonicotinic acid, the lowermost $\pi$ resonance lies within the gap, approximately $0.4 \mathrm{eV}$ further from the conduction-band edge than the comparable resonance in bi-isonicotinic acid. (b) Benzoic acid. For the calibration of the x-ray absorption spectrum, the ionization potential of the phenyl ring $\mathrm{C} 1 s$ level has been used. This procedure puts the peak ascribed to a $\mathrm{C}_{\text {carboxyl }} 1 s \rightarrow 3 b_{1}$ excitation at an ionization potential above the conduction band edge. If the ionization potential of the carboxylic $\mathrm{C} 1 s$ peak is used for calibration instead, this peak is found below the conduction band edge; cf. Fig. 5. (c) Sketch of the $3 b_{1}$ and $1 a_{2} \pi^{*}$ orbitals of $\mathrm{N} 1 s$-excited gas phase isonicotinic acid cut above the nodal plane (Ref. 30). These labels, which correspond to those for pyridine, are not strictly correct since the presence of the (nonsymmetrical) carboxylic group reduces the symmetry of isonicotinic acid with respect to pyridine. In the same way, these orbital labels are also used for the discussion of benzoic acid.

the photon energy scale is calibrated. This is done in the same manner as just described, by measuring a photoemission peak excited with first- and second-order light at an energy relevant for the XAS. The extremely high linearity and reproducibility of the monochromators in the present case then assures an accurate photon energy scale for the entire spectrum. The spectrum is then put onto the ionization potential scale as explained above.

The result is given in the uppermost curve of panel (c), in which the valence spectrum and the XAS were combined to show the adsorbate electronic structure. It has to be kept in mind that neither of these spectra correspond to the groundstate energies, but to the valence and core-excited ones, respectively. The shape of the former, however, often resembles the ground-state density of states quite closely (see, e.g., Ref. 19), as in the present case. In order to achieve a comparison to the substrate density of states, a clean crystal valence spectrum was measured and referenced to the vacuum level in the same way as described above (any other reference level would do as well, as discussed above). The lower curve in panel (c) gives this spectrum. The $\mathrm{O} 2 \mathrm{~s}$ and Ti $3 p$ levels at $27 \mathrm{eV}$ and $42 \mathrm{eV}$, respectively, align very well with those of the adsorbate spectrum. The unoccupied density of states has been included by giving an estimate for the location of the conduction-band edge, which is determined by the size of the band gap which has been set to $^{20}$ the optical value. $^{24}$

More sophisticated routes to a comparison of the adsorbate core-excited density of states to the substrate density of states comprise the inclusion of a substrate density-of-states calculation (this alternative was chosen in Ref. 5 for the biisonicotinic $\mathrm{acid} / \mathrm{TiO}_{2}(110)$ system) or of a properly referenced IPES measurement of the bare crystal. The latter pro- cedure has been used, for example, in Refs. 2 and 25, since it corresponds to the measurement of energies of electron traveling states and is therefore more reliable than ground-state theoretical results when electron-electron interactions are sizable. However, the acquisition of an angle-integrated spectrum which represents the density of states sufficiently well is not always achievable, as in the present case.

At this point it seems appropriate to give a comment on the use of the vacuum level. Ishii et al. ${ }^{26}$ have called attention to the fact that the local vacuum level is generally different from an idealized vacuum level at infinity because most surfaces are terminated by a dipole layer. We would like to stress that the method of obtaining the vacuum level presented here indeed determines its local value. The potential of a dipole layer with a (macroscopic, i.e., substrate diameter) extension $L$, where $L$ is typically in the range of millimeters, is essentially constant for observation distances from the sample much smaller $\operatorname{than}^{27}$ L. Hence, e.g., quantum tunneling does not play a role for the photoexcitation threshold. This implies, by virtue of energy conservation, that the secondary cutoff gives a measure of the local vacuum level. The vacuum level at infinity is in general not a measurable entity as spectrometer contact potentials influence the position of the vacuum level relevant for the photoelectron throughout its path to the detector. ${ }^{28}$ However, since the entire spectrum of an insulating sample shifts with the vacuum level (for cases of uniform potentials), the energy relationships determined using the local vacuum level are the correct ones. ${ }^{8}$

Now we would like to turn to two more applications of the procedure described. This concerns the monolayers of isonicotinic acid and of benzoic acid ${ }^{29}$ on rutile $\mathrm{TiO}_{2}(110)$; cf. Fig. 4. Isonicotinic acid is the monomer counterpart of 
bi-isonicotinic acid. Not unexpectedly, the electronic structure of $\mathrm{N} 1 s$-excited isonicotinic acid resembles quite closely that of bi-isonicotinic acid, with the lowest unoccupied orbital situated within the substrate bandgap [panel (a)]. The main difference is found for the higher unoccupied orbitals that overlap the conduction band. A comparison with benzoic acid excited at the ring carbon $K$ edge is now interesting, since this, by virtue of the $Z+1$ approximation, can be viewed as representative for the ground-state pyridinecarboxylic acid monomers (isonicotinic acid, nicotinic acid, and picolinic acid, all $\mathrm{C}_{6} \mathrm{H}_{4} \mathrm{NCOOH}$ ), although modified by the presence of an additional hydrogen atom bonded to the excited $\mathrm{C}$ atom. In the $Z+1$ approximation the atom on which the core hole produced in the XAS resides is replaced with an atom that possesses an additional nuclear charge. Such a description leads often to a quantitatively good description of the resonance energies in XAS. ${ }^{2,31}$ The coreexcited density of states of benzoic acid on $\mathrm{TiO}_{2}(110)$ is shown in panel (b) of Fig. 4. It is seen that the lowest absorption resonance now contains two spectral features which can be attributed to the $3 b_{1}$ and $1 a_{2}$ states $^{32}$ [cf. Fig. 4(c)]. For symmetry reasons the latter is not visible in the $\mathrm{N} 1 s$ XAS of isonicotinic acid or, at least, only as a slight broadening of the lowest resonance. From quantum chemical calculations it is expected that the lowest unoccupied orbital of isonicotinic acid overlaps the conduction band in the ground state, ${ }^{35}$ much as shown for bi-isonicotinic acid. ${ }^{36}$ Within the $Z+1$ approximation, this tendency is confirmed in Fig. 4 which shows that the lowest resonance of benzoic acid at 4.7 $\mathrm{eV}$ lies approximately $1 \mathrm{eV}$ closer to the vacuum level on that energy scale than the corresponding isonicotinic acid resonance. This confirms experimentally that the location of the lowest unoccupied state of isonicotinic acid within the band gap is largely an excitonic effect-i.e., due to the interaction between the core hole and excited electron.

Such effects are observed not only for core excitations, but for optical valence excitations as well. A priori the relationship between valence excitons and core excitons on the same molecules is not clear. A comparison of the binding energies of corresponding core and valence excitons in aromatic compounds $\left(\mathrm{C}_{60}\right.$, benzene, and pyridine) shows that these are very similar. ${ }^{37}$ This indicates that the core-level techniques employed here should have some impact in the interpretation of charge transfer in optically excited systems. Care has to be taken, however, to ensure that the excited states prepared with the different methods are comparable. The lowest excited state of "N3" (which contains biisonicotinic acid as a ligand; see the Introduction), for example, is a metal-to-ligand charge transfer (MLCT) state with no valence vacancy on the bi-isonicotinic acid ligand, ${ }^{38}$ and thus any excitonic effect could be expected to be significantly smaller in the organometallic system.

An analysis of the benzoic acid spectrum in Fig. 4(b) reiterates the importance of including the excitonic character in any analysis of the absorption spectra. The ionization potential of the carboxylic $\mathrm{C} 1 s$ orbital is about $4 \mathrm{eV}$ larger than that of the ring carbons. ${ }^{13}$ The existence of two distinct C $1 s$ absorption edges manifests itself in the XAS peak at $1.7 \mathrm{eV}$ in panel (b) of Fig. 4. It can be ascribed to an exci-

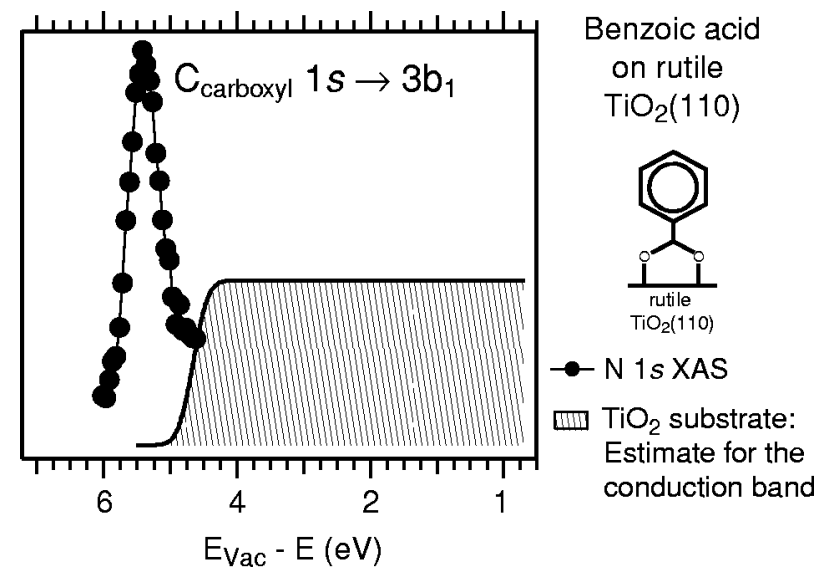

FIG. 5. Unoccupied density of states for benzoic acid for an XAS calibration which employs the ionization potential of the carboxylic $\mathrm{C} 1 s$. The only peak shown is that which is ascribed to a $\mathrm{C}_{\text {carboxyl }} 1 s \rightarrow 3 b_{1}$ excitation and which is found at approximately $1.7 \mathrm{eV}$ ionization potential in Fig. 4(b).

tation from the carboxylic $\mathrm{C} 1 \mathrm{~s}$ level to the lowest unoccupied orbital $\left(3 b_{1}\right) .{ }^{39}$ The same resonance is shown in Fig. 5 . Here, however, the carboxylic $1 s$ ionization potential was used for the energy scaling which places the resonance in the band gap. The position is actually closer in energy to that of $\mathrm{N} 1 s$ excited isonicotinic acid than for the phenyl C $1 s$ excited benzoic acid molecule. This illustrates that the corehole location has a direct impact on the electronic structure of the excited molecule and hence the size of the core exciton binding energy. ${ }^{40}$

Resonant photoemission can be used for the determination of whether an excited electron stays localized on the molecule during the core-hole lifetime or whether it is delocalized into a continuum coupled to the adsorbate. ${ }^{2}$ Without dwelling on the details of resonant photoemission we simply note that the occurrence of a resonant photoemission signal

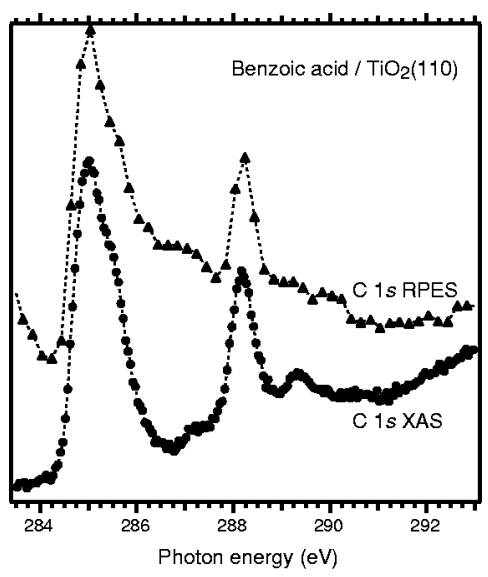

FIG. 6. Comparison of C $1 s$ XAS and RPES for benzoic acid. In both cases the spectra have been corrected for a highly varied higher-order background (Ref. 14). The strong resonant photoemission signals at $285 \mathrm{eV}$ and $288 \mathrm{eV}$ photon energy indicate strong localization of the excited electron to the adsorbate. An assignment of the smaller peaks is not motivated, as the background (Ref. 14) easily induces smaller structures. 
is a sign of full or partial localization during the core-hole lifetime (see, e.g., Refs. 5 and 41). A resonance within the substrate band gap efficiently prevents any transfer to the substrate on the femtosecond time scale, and thus a strong resonant photoemission signal should be observed. Similarly, a resonance in close proximity to the band edge could still show a relatively strong localization. ${ }^{42}$ Based on the total density of states shown in Fig. 4(b), a resonant photoemission signal is expected for both the $\mathrm{C}_{\text {phenyl }} 1 s \rightarrow 3 b_{1}$ and the $\mathrm{C}_{\text {carboxyl }} 1 s \rightarrow 3 b_{1}$ excitations, which is in good agreement $\mathrm{t}^{43}$ with the experimental observation in Fig. 6. Thus Fig. 6 is an experimental corroboration of the assignment of the resonance in Fig. 5.

\section{CONCLUSIONS}

It has been detailed how photoemission and x-ray absorption spectra together with an estimation for the substrate density of states can be employed to construct a full image of the core-excited density of states for an adsorbate on a semiconductor surface. This concerns in particular the relationship between the core-excited unoccupied density of states of the adsorbate in comparison to the unoccupied states of the substrate. The procedure thus allows one to assess the electronic matching between adsorbate and substrate.

The alignment procedure has been applied to three cases: namely, bi-isonicotinic acid, isonicotinic acid, and benzoic acid adsorbed on rutile $\mathrm{TiO}_{2}(110)$. It has been seen that coreexcitonic effects play an important role in the exact energy relationships and that these effects depend on the location of the core hole. Since similar (valence) excitonic effects may occur in optically excited samples, ${ }^{37}$ the techniques used here can, when employed with care, also be used to gain information on systems in which (visible) light absorption plays an important role.

\section{ACKNOWLEDGMENT}

We would like to acknowledge K. Hansen, S. Wiklund, R. Nyholm, and M. Leandersson for help with beamline D1011 and our funding sources Vetenskapsrådet, Göran Gustafssons Stiftelse, and the CARAMEL Consortium, which is funded by Stiftelsen för Strategisk Forskning.

${ }^{13}$ J. Schnadt, J. N. O’Shea, L. Patthey, J. Schiessling, J. Krempasky, M. Shi, N. Mårtensson, and P. A. Brühwiler (unpublished).

${ }^{14}$ J. Schnadt, J. Schiessling, J.N. O'Shea, L. Patthey, M. Shi, C. Puglia, N. Mårtensson, and P.A. Brühwiler, Nucl. Instrum. Methods Phys. Res. B 184, 609 (2001).

${ }^{15}$ A. Hagfeldt and M. Grätzel, Acc. Chem. Res. 33, 269 (2000).

${ }^{16}$ S. Hüfner, Photoelectron Spectroscopy, 2nd ed. (Springer, Berlin, 1996), Chap. 2.

${ }^{17}$ The effects of charging on the spectra energies can be minimized by choosing the sample and detection geometries accordingly. We chose normal emission to maintain a cylindrical symmetry of the field lines between the biased circular sample and conical analyzer lens; much of the signal excited at the sample surface is lost for increasing emission angle, which is especially critical when measuring the secondary electrons. Any comparison measurements were made in quick succession, to avoid possible light intensity changes, which can in principle affect the sample charging rate, and thus the steady state charge.

${ }^{18}$ The analyzer settings are an issue only if different settings shift the binding energy scale, which is the present case.

${ }^{19}$ S. Hüfner, Photoelectron Spectroscopy, 2nd ed. (Springer, Berlin, 1996), pp. $353 \mathrm{ff}$.

${ }^{20}$ This choice for the band gap can be justified by the following arguments. The substrate density of states relevant for transport properties, as well as spectroscopy, is one that contains an extra quasiparticle (electron or hole). Principally, this corresponds to a density of states obtained in a quasiparticle calculation which contains self energy corrections on position and bandwidth (Ref. 21). We have found, however, that a calculated ground-state density of states models the valence band very well in the present case (Ref. 5), which suggests that also the band gap (and the unoccupied states) can be modeled by a ground-state value. Similarly, self-energy corrections are negligible, for example, in the description of the XAS of graphite (Ref. 22). The optical gap value is indeed a good measure of the ground-state band gap, for 
the present case, since no electronic excitations at energies less than the band gap occur for $\mathrm{TiO}_{2}$ (Ref. 23).

${ }^{21}$ E.L. Shirley, Phys. Rev. B 58, 9579 (1998).

${ }^{22}$ R. Ahuja, P.A. Brühwiler, J.M. Wills, B. Johansson, N. Mårtensson, and O. Eriksson, Phys. Rev. B 54, 14396 (1996).

${ }^{23}$ V. E. Henrich and P. A. Cox, The Surface Science of Metal Oxides (Cambridge University Press, Cambridge, England, 1994).

${ }^{24}$ D.C. Cronemeyer, Phys. Rev. 87, 876 (1952).

${ }^{25}$ R. Schwedhelm, L. Kipp, A. Dallmeyer, and M. Skibowski, Phys. Rev. B 58, 13176 (1998).

${ }^{26}$ H. Ishii, K. Sugizama, E. Ito, and K. Seki, Adv. Mater. (Weinheim, Ger.) 11, 605 (1999).

${ }^{27}$ An estimate of over which range the potential is essentially constant can be derived as follows: assuming a circular shaped sample with radius $L / 2 \approx 0.5 \mathrm{~cm}$ with a constant dipole layer across the sample, a geometry which is quite typical for a PES experimental arrangement, the potential on the symmetry axis is proportional to $-\cos (\arctan L / 2 d)+1$ with $d$ being the distance from the sample. For $d=5 \times 10^{-5} \mathrm{~m}$ the potential has not decreased by more than $1 \%$. This is much larger than the range over which quantum tunneling could allow the experiment to probe a "nonlocal" vacuum level. It should be noted, however, that the dipole layer potential decreases to approximately half its central axis value when the observation point is chosen at the same small distance $d$ from the sample, but lying on a line that is perpendicular to the sample and that cuts it at the edge.

${ }^{28}$ R.T. Poole, R.C.G. Leckey, J.G. Jenkin, and J. Liesegang, J. Phys. E 6, 201 (1973).

${ }^{29}$ Due to analyzer problems, it was not possible to carry out the vacuum-level calibration procedure for the isonicotinic acid and benzoic acid samples. Instead, they were aligned at the $\mathrm{O} 1 \mathrm{~s}$ substrate peak of the bi-isonicotinic acid case. As outlined in the text, this procedure does not affect the validity of the resulting image of the electronic structure. For isonicotinic acid the results obtained in this manner are consistent with an alignment at the Ti $3 p$ substrate level to within $0.05 \mathrm{eV}$, while for benzoic acid the results differed by not more than $0.1 \mathrm{eV}$.

${ }^{30} \mathrm{M}$. Odelius (private communication).
${ }^{31}$ A. Sandell, O. Hjortstam, A. Nilsson, P.A. Brühwiler, O. Eriksson, P. Bennich, P. Rudolf, J.M. Wills, B. Johansson, and N. Mårtensson, Phys. Rev. Lett. 78, 4994 (1997).

${ }^{32}$ This assignment is supported by the gas phase measurements and calculations of Ref. 33. Here as well as in Ref. 34 it was found that the $\mathrm{C}_{\text {phenyl }} 1 s \rightarrow 3 b_{1}$ excitation splits up into two components due to the chemical shift between the $\mathrm{C}$ atoms of the phenyl ring. In addition, Kolczewski et al. (Ref. 33) observe an additional peak that is ascribed to a $\mathrm{C}_{\text {phenyl }} 1 s \rightarrow 1 a_{2}$ excitation. We argue that the shoulder observed in the present experiment is due to the latter excitation, while the former is not resolved into two peaks. The argument is based upon the finding that the XAS peak at $4.8 \mathrm{eV}$ ionization potential is much larger in intensity than the structure at $4.2 \mathrm{eV}$ ionization potential, while the intensity ratio between the two $\mathrm{C}_{\text {phenyl }} 1 s \rightarrow 3 b_{1}$ structures should amount to approximately (Ref. 33) 2:3.

${ }^{33}$ C. Kolczewski et al., J. Chem. Phys. 115, 6426 (2001).

${ }^{34}$ C. Hannay, D. Duflot, J.-P. Flament, and M.-J. Hubin-Franskin, J. Chem. Phys. 110, 5600 (1999).

${ }^{35}$ P. Persson, S. Lunell, and L. Ojamäe, Chem. Phys. Lett. 364, 469 (2002).

${ }^{36}$ P. Persson et al., J. Chem. Phys. 112, 3945 (2000).

${ }^{37}$ J. Schnadt, J. Schiessling, and P. A. Brühwiler (unpublished).

${ }^{38}$ M.K. Nazeeruddin, A. Kay, I. Rodicio, R. Humphry-Baker, E. Müller, P. Liska, N. Vlachopoulos, and M. Grätzel, J. Am. Chem. Soc. 115, 6382 (1993).

${ }^{39}$ M. Neuber, M. Zharnikov, J. Walz, and M. Grunze, Surf. Rev. Lett. 6, 53 (1999).

${ }^{40}$ A.J. Maxwell, P.A. Brühwiler, D. Arvanitis, J. Hasselström, and N. Mårtensson, Phys. Rev. Lett. 79, 1567 (1997).

${ }^{41}$ J. Schnadt et al. (unpublished).

${ }^{42}$ S. Ramakrishna, F. Willig, and V. May, J. Chem. Phys. 115, 2743 (2001).

${ }^{43}$ If the transition were instead from the phenyl C $1 s$ level, then the resonance at $288 \mathrm{eV}$ would have been degenerate with the substrate conduction band, and fast charge transfer would be expected, quenching any resonant photoemission signal as in Refs. 5 and 41. 